\title{
Increased expression of PD-L1 by the human papillomavirus 16 E7 oncoprotein inhibits anticancer immunity
}

\author{
CHAOQI LIU ${ }^{1 *}$, JIAO LU $^{1,2^{*}}$, HUIQUN TIAN $^{1}$, WEI DU ${ }^{1,2}$, \\ LIN ZHAO $^{1,2}$, JING FENG ${ }^{1,2}$, DING YUAN ${ }^{2}$ and ZHIYING LI $^{2}$ \\ ${ }^{1}$ Hubei Key Laboratory of Tumor Microenvironment and Immunotherapy; \\ ${ }^{2}$ The Second Clinical Medical College, China Three Gorges University, Yichang, Hubei 443001, P.R. China
}

Received October 21, 2015; Accepted November 11, 2016

DOI: $10.3892 / \mathrm{mmr} .2017 .6102$

\begin{abstract}
Cytotoxic T lymphocyte dysfunction is frequently associated with PD-L1/PD-1 pathway activation, and is a principal obstacle in cancer therapy. In the present study, the mechanisms underlying the human papillomavirus (HPV)-induced evasion of cervical cancer cells to the host immune system via the programmed death ligand 1/programmed death 1 (PD-L1/PD-1) signaling pathway was investigated. A significant increase in the expression of the HPV16E7 viral protein and PD-L1 in cervical tissues was observed when compared with normal cervical tissues. In addition, a positive correlation between HPV16E7 and PD-L1 expression was observed by immunohistochemical staining and reverse transcription-polymerase chain reaction. Overexpressing HPV16E7 oncoprotein in the epithelial carcinoma of PC3 cells increased the expression level of the PD-L1 protein and inhibited peripheral blood mononuclear cell (PBMC) proliferation and cytotoxic T lymphocyte (CTL) activity. Upon knockdown of HPV16E7 in HPV16-associated CaSki cervical cancer cells with a relevant siRNA, a reduction in PD-L1 protein expression was observed, as well as a significant increase in PBMC proliferation and CTL activity. A recombinant plasmid, MSCVPIG-soluble PD-1, was constructed and transfected into the CaSki cell line, and was co-cultured with PBMCs. PBMC proliferation and CTL activity were observed to increase significantly. In conclusion, the results presented in the current study suggest that overexpression of PD-L1, induced by HPV16E7, may be responsible
\end{abstract}

Correspondence to: Professor Ding Yuan or Professor Zhiying Li, The Second Clinical Medical College, China Three Gorges University, 410 Yiling Avenue, Yichang, Hubei 443001, P.R. China

E-mail: yxyydyd@126.com

E-mail: lzying1968@163.com

\section{*Contributed equally}

Key words: cervical cancer, E7 oncoprotein, human papillomavirus 16 , programmed death-ligand 1 , soluble programmed death ligand 1 for lymphocyte dysfunction. In addition, soluble PD-1 may restore the function of tumor-infiltrating lymphocytes by inhibiting the PD-L1/PD-1 signaling pathway. These results may provide a novel insight for immunotherapeutic approaches in the treatment of cervical cancer.

\section{Introduction}

Cervical cancer is one of the most common gynecological malignancies, with 500,000 new cases diagnosed annually worldwide, and approximately one-third of these cases leading to mortality (1). High-risk human papillomavirus (HPV) types, such as HPV16, 18 and 31 are associated with $>90 \%$ of cervical cancer cases (2). The high-risk HPV oncoproteins, E6 and E7, contribute to cervical carcinogenesis through inactivation of the cellular tumor suppressor proteins p53 (3) and retinoblastoma (4), respectively. The current treatments for cervical cancer are comprehensive and include surgery, radiotherapy, chemotherapy and immunotherapy. Previous studies have predicted that immunotherapy may be a promising strategy as it has a record of feasibility and safety in the treatment of cancer $(5,6)$. However, cytotoxic T lymphocyte dysfunction is the principal obstacle for cancer immunotherapy. The negative co-stimulating signaling molecules in the programmed death ligand 1/programmed death 1 (PD-L1/PD-1) signaling pathway have been previously correlated with tumor-immune escape (7). The interaction between PD-L1 on antigen-presenting cells (APCs) and its receptor PD-1 on T lymphocytes, leads to inhibition of $\mathrm{T}$ lymphocyte activation and induction of apoptosis or anergy of T lymphocytes $(8,9)$. Aberrant high expression of PD-L1 is common in various tumors and has been correlated with tumor progression and patient survival $(10,11)$. The aim of the present study was to investigate whether HPV oncoprotein-induced cervical epithelial cells circumvent the host immune system via the PD-L1/PD-1 signaling pathway.

\section{Materials and methods}

Cell culture and transfection. The HPV16-associated cervical cancer cell line, CaSki, and a HPV-negative epithelial cell line, PC3, were obtained from the China Center for Type Culture Collection (Wuhan, China). Cells were cultured in RPMI-1640 medium (Gibco; Thermo Fisher Scientific, Inc., Waltham, MA, 
USA) supplemented with $10 \%$ newborn calf serum (NBCS; Gibco; Thermo Fisher Scientific, Inc.) and 1\% penicillin/streptomycin (North China Pharmaceutical Co., Ltd., Hebei, China) at $37^{\circ} \mathrm{C}$ in $5 \% \mathrm{CO}_{2}$. Purified plasmids MSCVPIG (Addgene, Cambridge, MA, USA) and MSCVPIG-SPD-1 (constructed in the lab) were stably transfected into PC3 cells $\left(1 \times 10^{5} / \mathrm{ml}\right)$ using Lipofectamine 2000 (Invitrogen; Thermo Fisher Scientific, Inc.) according to the manufacturer's instructions. After 2 weeks, positive clones were selected based on their resistance to G418 (800 mg/ml; North China Pharmaceutical Co., Ltd.), which were analyzed by reverse transcription-polymerase chain reaction (RT-PCR), flow cytometry and western blot analysis.

Cell viability assay. Stable transfection clones were seeded into 96 -well plates $\left(1 \times 10^{4}\right.$ cells/well $)$ and cultured in RPMI-1640 medium supplemented with 10\% NBCS and 1\% penicillin/streptomycin at $37^{\circ} \mathrm{C}$ and $5 \% \mathrm{CO}_{2}$. MTT reagent ( $5 \mathrm{mg} / \mathrm{ml}$; Sigma-Aldrich; Merck Millipore, Darmstadt, Germany) was added to the cells at different time-points $(12,24,48,72$ and $96 \mathrm{~h})$ and were incubated for $4 \mathrm{~h}$. After discarding the supernatant, $100 \mu 1$ dimethyl sulfoxide was added to each well and the plates were agitated at $37^{\circ} \mathrm{C}$ for $15 \mathrm{~min}$. The absorbance of samples was recorded at $490 \mathrm{~nm}$ using a Multiskan Spectrum (Thermo Fisher Scientific, Inc.).

Flow cytometry. PC 3 cells $\left(0.5-1 \times 10^{6}\right)$ were collected, washed with $1 \mathrm{X}$ phosphate-buffered saline (PBS) and fixed in $4 \%$ paraformaldehyde at $4^{\circ} \mathrm{C}$ for $30 \mathrm{~min}$, and then incubated with $0.1 \%$ Triton X-100 (Sigma-Aldrich; Merck Millipore), containing $2 \%$ bovine serum albumin (BSA Sigma-Aldrich; Merck Millipore) at room temperature for $15 \mathrm{~min}$. After blocking with $2 \% \mathrm{BSA}$ at $37^{\circ} \mathrm{C}$ for $30 \mathrm{~min}$, the cells were incubated with mouse anti-HPV16E7 (cat no. sc-6981) or rabbit anti-human PD-L1 (cat no. sc-50298) primary antibodies (dilution 1:1,000; Santa Cruz Biotechnology, Inc., Dallas, TX, USA) for $30 \mathrm{~min}$ at $37^{\circ} \mathrm{C}$, and then incubated with either fluorescein isothiocyanate (FITC)-conjugated donkey anti-mouse (cat. no. 715-005-151) or goat anti-rabbit (cat. no. 111-095-003) secondary antibodies (dilution 1:3,000; Jackson ImmunoResearch Laboratories, Inc., West Grove, PA, USA) for $30 \mathrm{~min}$ at room temperature. Cells were resuspended in $500 \mu 1 \mathrm{1X}$ PBS and analyzed by fluorescence-activated cell sorting to determine the expression levels of E7 and PD-L1. The same protocol was used, with the exception of the use of an isotype control (cat. no. sc-2025 or sc-2027; Santa Cruz Biotechnology, Inc.) in place of the primary antibodies.

In order to perform cell cycle analysis, PC3 cells were collected, washed with 1X PBS and fixed with 70\% ethanol for $1 \mathrm{~h}$ at $4^{\circ} \mathrm{C}$. The cells were then treated with $0.1 \%$ Triton X-100 and $50 \mu \mathrm{g} / \mathrm{ml}$ RNase (Sigma-Aldrich; Merck Millipore), and labeled with $20 \mu \mathrm{g} / \mathrm{ml}$ propidium iodide (Sigma-Aldrich; Merck Millipore) for $30 \mathrm{~min}$ at room temperature. A total of $1 \times 10^{4}$ cells were analyzed for each sample with flow cytometry using the Coulter EPICS flow cytometer (Beckman Coulter, Inc., Brea, CA, USA).

$R T-P C R$. PC3 cells $\left(1 \times 10^{6}\right)$ were collected and the total RNA was extracted using TRIzol reagent (Invitrogen; Thermo Fisher Scientific, Inc.) and cDNA was synthesized using the
SuperScript ${ }^{\mathrm{TM}}$ II reverse transcriptase (Invitrogen; Thermo Scientific, Inc.) at $65^{\circ} \mathrm{C}$ for $5 \mathrm{~min}, 42^{\circ} \mathrm{C}$ for $1 \mathrm{~h}$ and $70^{\circ} \mathrm{C}$ for $5 \mathrm{~min}$, according to the manufacturer's instructions. PCR amplification was performed using specific oligonucleotide primers (Sangon Biotech Co., Ltd., Shanghai, China). $\beta$-actin was used as an endogenous control. HPV16E7 forward, 5'-GCT CAGAGGAGGAGGATGAAATAGA-3' and reverse, 5'-CAC AACCGAAGCGTAGAGTCAC-3'; PD-L1 forward, 5'-GCTA TGGTGGTGCCGACTACA-3' and reverse, 5'-CTTGATGGT CACTGCTTGTCCA-3'; $\beta$-actin forward, 5'-CTAAGTCATA GTCCGCCTAGAAGCA-3' and reverse, 5'-CTAAGTCATAG TCCGCCTAGAAGCA-3'. PCR reactions contained $25 \mathrm{ng}$ cDNA template, $100 \mathrm{ng}$ sense and antisense oligonucleotide primers, $2.5 \mu 1$ Taq PCR buffer (Promega Corporation, Madison, WI, USA), $0.4 \mathrm{mM}$ dNTP mixture, and 1U Taq polymerase (Promega Corporation, Madison, WI, USA) in a total reaction volume of $25 \mu \mathrm{l}$. Following an initial $3 \mathrm{~min}$ incubation step at $94^{\circ} \mathrm{C}$, PCR was performed using 30 cycles of denaturation at $94^{\circ} \mathrm{C}$ for $30 \mathrm{~s}$, annealing at $55^{\circ} \mathrm{C}$ for $30 \mathrm{~s}$ and elongation at $72^{\circ} \mathrm{C}$ for $30 \mathrm{~s}$. PCR products were separated by electrophoresis at $100 \mathrm{~V}$ for 40 min using a $2 \%$ agarose gel, and were detected by ethidium bromide staining.

Western blotting. CaSki cell $\left(5 \times 10^{6}\right)$ were collected and lyzed in 1X SDS sample buffer containing 5\% $\beta$-mercaptoethanol (Sigma-Aldrich; Merck Millipore) at $4^{\circ} \mathrm{C}$. The protein samples were denatured by heating at $95^{\circ} \mathrm{C}$ for $5 \mathrm{~min}$, and $30 \mu \mathrm{g} / \mathrm{well}$ protein was separated in a $12 \%$ SDS-PAGE Bis-Tris gel (Invitrogen; Thermo Fisher Scientific, Inc.) in 1X MES SDS running buffer (Invitrogen; Thermo Fisher Scientific, Inc.), and then transferred to a polyvinylidene difluoride (PVDF) membrane (current, $250 \mathrm{~mA}$; duration, $2 \mathrm{~h}$ ). The PVDF membrane was blocked with 5\% non-fat milk in Tris-buffered saline with $0.1 \%$ Tween-20 (TBST; Sigma-Aldrich; Merck Millipore) at $4^{\circ} \mathrm{C}$ overnight. After rinsing with TBST, the membrane was incubated at room temperature for $2 \mathrm{~h}$ with a primary rabbit anti-human PD-1 antibody (cat no. sc-10295; Santa Cruz Biotechnology, Inc.) at 1:500 dilution in TBST and then washed three times with TBST. Proteins were incubated with horseradish peroxidase-conjugated goat anti-rabbit secondary antibody at 1:3,000 dilution in TBST for $1 \mathrm{~h}$ at room temperature, before washing three times with TBST. The immunoreactive proteins were detected with a chemiluminescence substrate (Pierce; Thermo Fisher Scientific, Inc.), and the signals were captured on an X-ray film in a darkroom. The relative protein level in each sample presented in a bar graph was calculated based on the protein band density after normalizing to $\beta$-actin (cat. no. sc-47778; Santa Cruz Biotechnology, Inc.) (1:1,000 dilution in TBST) for sample loading.

Co-culture and lymphocyte proliferation. Peripheral blood mononuclear cells (PBMCs) were isolated from the blood of 20 healthy donors (Center Blood Bank of Yichang City, Yichang, China) by density gradient centrifugation (536 x g for $20 \mathrm{~min}$ ) over Ficoll/Hypaque (Sigma-Aldrich; Merck Millipore), according to the manufacturer's instructions. PBMCs were resuspended to a final concentration of $1 \times 10^{6} / \mathrm{ml}$ in PBS with $0.1 \%$ BSA, before they were stained with $5 \mu \mathrm{mol} / 1$ 5-(-6)-carboxyfluorescein diacetate succinimidyl ester (CFSE; Molecular Probes; Thermo Fisher Scientific, Inc.) for $10 \mathrm{~min}$ 
at $37^{\circ} \mathrm{C}$. The stain was quenched by incubating cells for $5 \mathrm{~min}$ in PBS containing $10 \%$ fetal bovine serum. Excess CFSE was removed by adding PBS and centrifuging (134 x g for $5 \mathrm{~min}$ ). This wash-step was repeated twice. The CFSE-labeled PBMCs were co-cultured with stable transfection clone sPD1-PC3 cells at a ratio of 1:100. Following 2 days of culture, the PBMCs were harvested and washed three times, resuspended with $300 \mu \mathrm{l}$ PBS, subjected to FACS analysis $(\mathrm{Ex} / \mathrm{Em}=488 / 518 \mathrm{~nm})$, and analyzed using CellQuest Pro software version 5.1 (BD Biosciences, San Diego, CA, USA) to determine the cell proliferation.

Lymphocyte cytotoxicity assay. Using above PBMCs stimulated with tumor cells serving as effector cells, the target cells (1x $10^{4} /$ well) were incubated at different effector-to-target ratios (1:1, 50:1 and 100:1). The following three control groups were established: PBMCs with 1\% NP-40 (Sigma-Aldrich; Merck Millipore), denoted as the 'maximum lytic group'; target cells, denoted as the 'effector spontaneous release group'; and the remaining group containing phenolsulfonphthalein medium as the 'blank control group'. All of the cells were incubated at $37^{\circ} \mathrm{C}$ for $6 \mathrm{~h}$. Centrifuged supernatant $(536 \mathrm{x} \mathrm{g}$ for $10 \mathrm{~min} ; 50 \mu \mathrm{l}$ ) was collected to assess the quantity of lactate dehydrogenase by quantitative measurements using the Cytotox 96 Non-Radioactive Cytotoxicity assay kit (Promega Corporation) according to the manufacturer's instructions. The lytic activity of lymphocytes was assessed using the mean value from triplicate repeats according to the following formula: Percentage of specific lysis $=($ experimental release - spontaneous release)/(maximum release - spontaneous release x100.

Luciferase reporter gene assay. PC3 cells $\left(1 \times 10^{5} / \mathrm{ml}\right)$ were transiently transfected with the PD-L1-firefly luciferase reporter gene generated at the Institute of Molecular Biology of China Three Gorges University (Yichang, China). Following $48 \mathrm{~h}$ of transfection, luciferase activity was measured using the Dual-Luciferase ${ }^{\circledR}$ Reporter assay system (E1910, Promega Corporation) according to the manufacturer's instructions. Luminescence was read from the 96-well plate using an Infinite $^{\mathrm{TM}} 200$ luminometer (Tecan, Männedorf, Switzerland).

Histopathological analysis and immunohistochemical staining. Cervical carcinoma (40 patients) and normal cervical tissue ( 8 individuals) samples were obtained from the Department of Obstetrics and Gynecology, the Second Affiliated Hospital of China Three Gorges University (Yichang, China) between March 2010 and May 2011. All patients did not accept any pretreatment for six months prior to the study. The study met the criteria of the Ethics Committee of The Second Affiliated Hospital of China Three Gorges University (Yichang, China). All cervical tissue specimens were fixed with $10 \%$ neutral formalin, embedded with paraffin, and serially-sectioned at $5 \mu \mathrm{m}$. The sections were mounted onto the histostick-coated slides. A total of 4-5 adjacent sections were collected for histopathological analysis and immunohistochemical staining. The clinical histopathological diagnosis of all cases was performed according to the WHO (2003) standard (12).

The biotin-streptavidin complex kit (Boshide Biological engineering Ltd., Wuhan, China) was used for the
Table I. PD-L1 and HPV16E7 expression in normal cervical and cervical cancer tissues.

\begin{tabular}{lrcc}
\hline & $\mathrm{n}$ & PD-L1 (\%) & HPV16E7 (\%) \\
\hline Normal control & 8 & $0(0)$ & $1(12.5)$ \\
Cervical carcinoma & 40 & $38(95)^{\mathrm{a}}$ & $33(82.5)^{\mathrm{a}}$ \\
\hline
\end{tabular}

${ }^{a} \mathrm{P}<0.05$ vs. normal control. PD-L1, programmed death-ligand 1 ; HPV16E7, human papillomavirus 16 E7 oncoprotein. The expression levels of these proteins were quantified by immunohistochemical staining using Image-Pro Plus 6.0 software.

immunostaining of PD-L1 and HPV16E7. All of the following agents were part of the kit, except the primary antibodies. According to the manufacturer's protocol the procedures were briefly as follows: Following de-waxing, inactivation of endogenous peroxidase activity and inhibition of cross-reactivity using normal non-immune goat serum, the sections were incubated at $4^{\circ} \mathrm{C}$ overnight with a diluted solution of the primary antibodies (as aforementioned). Localization of the primary antibodies was achieved by subsequent application of a biotin-conjugated IgG secondary antibody, and a streptavidin-conjugated peroxidase. Signals were visualized with diaminobenzidine, and cellular nuclei were counterstained with instant hematoxylin. Negative controls were established by replacing the primary antibody with normal isotype control (the kit supplied). The quantity of positive staining was analysed as follows: Opening the image analysis system, defining the negative and positive standard, followed by scanning the samples. The relative brightness values were obtained using the Image-Pro Plus 6.0 software (Media Cybernetics, Washington DC, USA).

Statistical analysis. Data were analyzed using SPSS software (version 13.0; SPSS, Inc., Chicago, IL, USA). Data are expressed as the mean \pm standard deviation. Differences between two groups were analyzed with Student's t-test or chi-square test. $\mathrm{P}<0.05$ and $\mathrm{P}<0.01$ were considered to indicate statistically significant differences.

\section{Results}

Expression of HPV16E7 and PD-L1 in cervical tissues was positively correlated. In order to explore the correlation between HPV16E7 and PD-L1 expression, the present study examined their mRNA and protein expression levels in clinically diagnosed cervical cancer tissues. Immunohistochemical analysis demonstrated that the normal cervical epithelium rarely expressed PD-L1, while the majority of cervical cancer samples exhibited strong expression of PD-L1 ( $\mathrm{P}=0.017$; Table I). HPV16E7 expression was detected using immunohistochemistry and RT-PCR analyses. The results demonstrated that HPV16E7 was expressed at low levels in normal tissues and at high levels in cervical cancer tissues, with HPV16E7 protein primarily localized to the nucleus and cytoplasm of the cervical cancer cells (Fig. 1). Statistical analysis of these data indicated that HPV16E7 and PD-L1 protein expression was positively correlated $(r=0.531)$ 

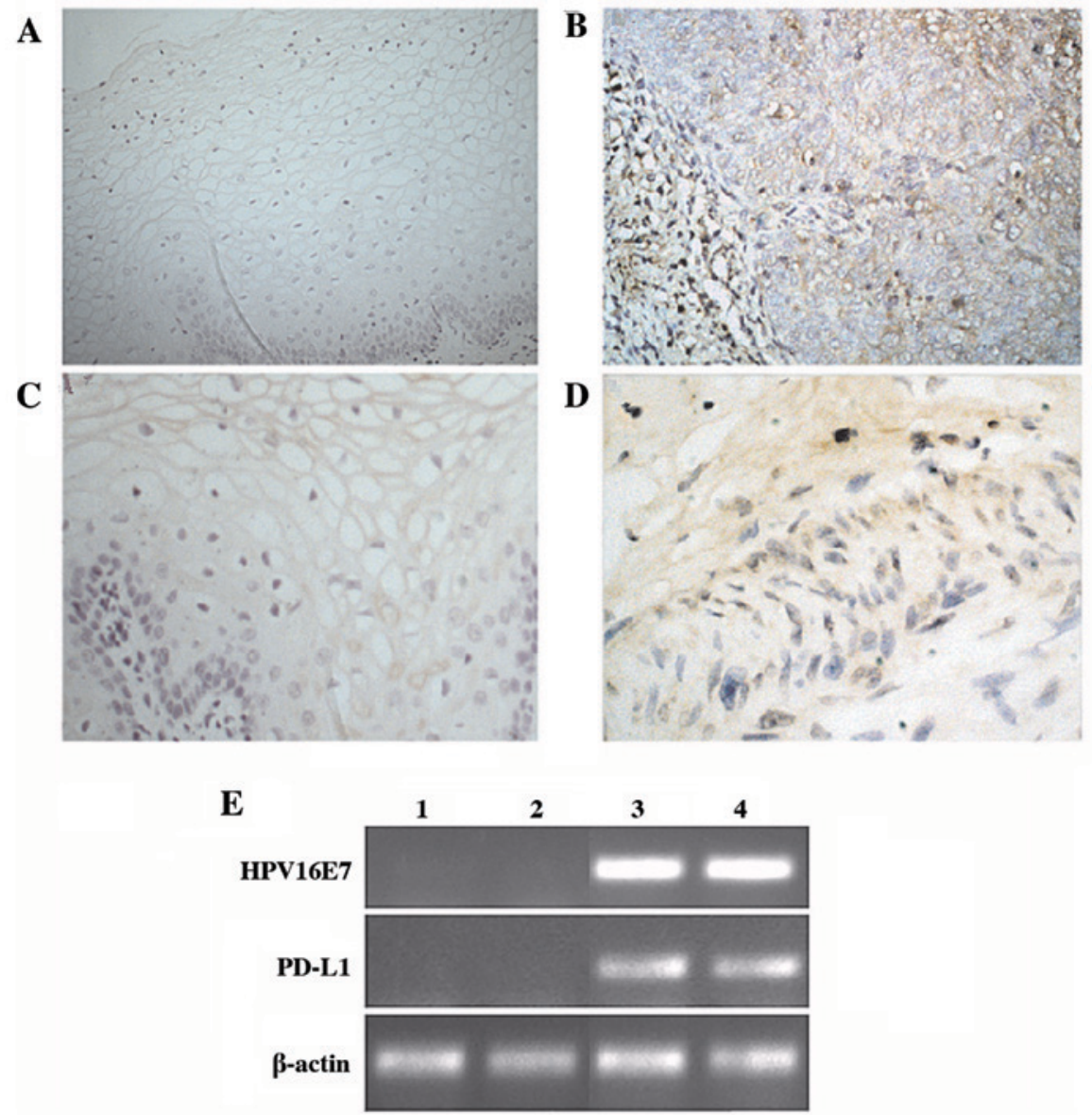

Figure 1. Expression of HPV16E7 and PD-L1 in cervical tissues. Immunohistochemical staining and RT-PCR analyses were used to detect the protein and mRNA levels of HPV16E7 and PD-L1. Representative images of HPV16E7 staining in (A) normal cervical tissues and (B) cervical cancer tissues (magnification, $\mathrm{x} 40$ ). Representative images of PD-L1 staining in (C) normal cervical tissues and (D) cervical cancer tissues (magnification, $\mathrm{x} 40$ ). (E) mRNA expression levels of HPV16E7 and PD-L1, as detected by RT-PCR analysis. Lanes 1 and 2, normal cervical tissues; lanes 3 and 4, cancerous cervical tissues. HPV16E7, human papillomavirus 16 E7 oncoprotein; PD-L1, programmed death-ligand 1; RT-PCR, reverse transcription-polymerase chain reaction.

in cervical cancer tissues and reached statistical significance $(\mathrm{P}=0.043)$.

HPV16E7 upregulated PD-L1 expression and inhibited lymphocyte activity. The expression of HPV16E7 and PD-L1 demonstrated a positive correlation in cervical carcinoma tissues. In order to further explore this correlation, a PC 3 cell line stably expressing HPV16E7 (PC3-E7) was established by transfecting the pcDNA3.1 (-)-E7 plasmid or an empty vector control (PC3-3.1), and the expression levels of PD-L1 were measured. PC3-E7 clones demonstrated the oncogenic characteristics of enhanced viability, a significant reduction in the number of cells at the G0/G1 phase, an accumulation of cells at $\mathrm{S}$ phase, and no obvious difference at $\mathrm{G} 2 / \mathrm{M}$ phase when compared with the empty vector controls (Fig. 2A-C). Flow cytometry analysis and a luciferase reporter system were used to detect PD-L1 expression in order to investigate whether PD-L1 expression may be regulated by HPV16E7. The results demonstrated that PD-L1 was overexpressed in the PC3-E7 clones when compared with the empty vector control cells (Fig. 2D). The luciferase assay results demonstrated that fluorescence values in the PC3-E7 cells were significantly higher than the control cells $(\mathrm{P}=0.022$; Fig. $2 \mathrm{E})$. These results suggest that HPV16E7 promoted PD-L1 expression in PC3-E7 cells. PD-L1 expressed on the surface of tumor cells inhibits lymphocyte activity through binding to PD-1 on lymphocytes (13). Consistent with these observations, the proliferation rate of lymphocytes was significantly reduced following co-culture with PC3-E7 cells compared with PC3-3.1 cells (Fig. 2F and G).

In order to further investigate the correlation between HPV16E7 and PD-L1, HPV16E7 was knocked down using RNA interference in the CaSki cell line, which had undergone integration of HPV16 genes, and PD-L1 expression levels were detected. A pSilencer ${ }^{\mathrm{TM}}$ 2.1-U6-E7siRNA plasmid was constructed and stably transfected into CaSki cells. Positive clones were identified by flow cytometry and denoted as CaSki-E7si cells. Empty vector control-transfected cells were denoted as CaSki-si-ctl. Suppression of HPV16E7 expression in CaSki-E7si cells was confirmed by flow cytometry analysis (Fig. 3A). The viability of CaSki-E7si cells was significantly suppressed compared with the controls [ $\mathrm{P}=0.041(48 \mathrm{~h})$; $\mathrm{P}=0.028$ (72 h); P=0.008 (96 h); Fig. 3B]. Flow cytometry analysis of cell cycle was conducted subsequent to E7 siRNA treatment. It was demonstrated that the cell cycle distribution was altered in the CaSki-E7si cells. The percentage of cells in the sub-G1 phase increased from $2.1 \%$ in the untreated control to $33.6 \%$ following E7 siRNA expression ( $\mathrm{P}=0.018$; 
A
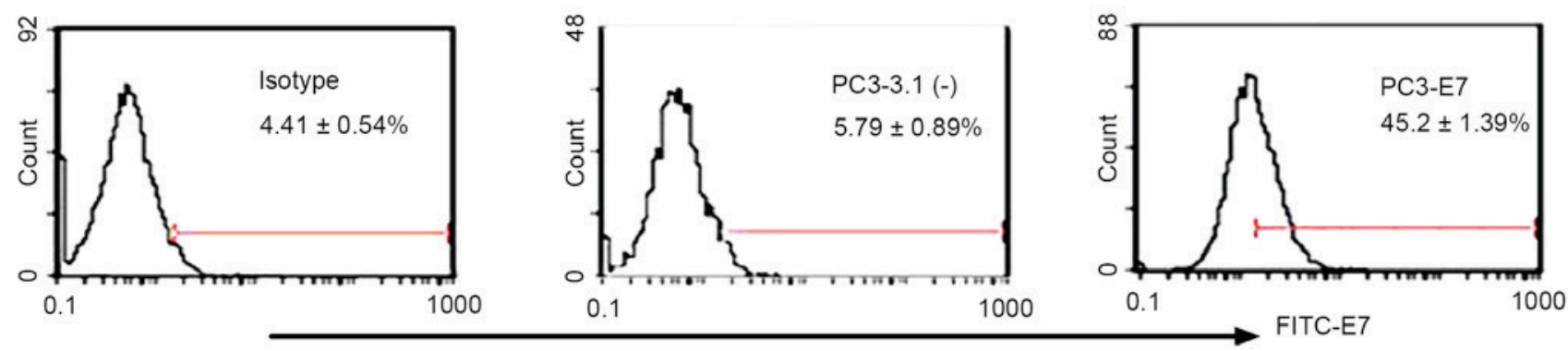

B

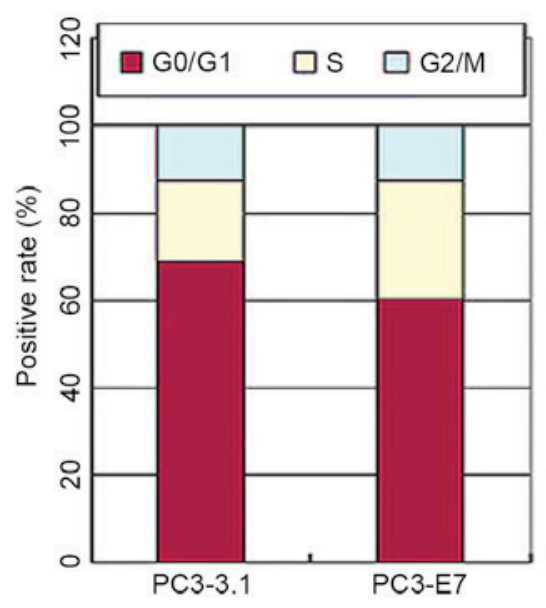

$\mathbf{E}$

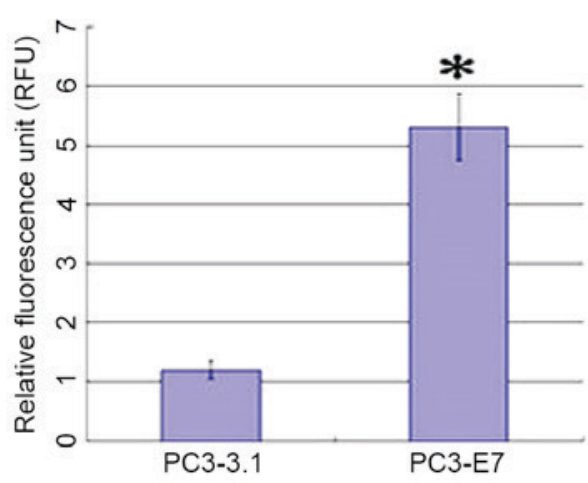

C

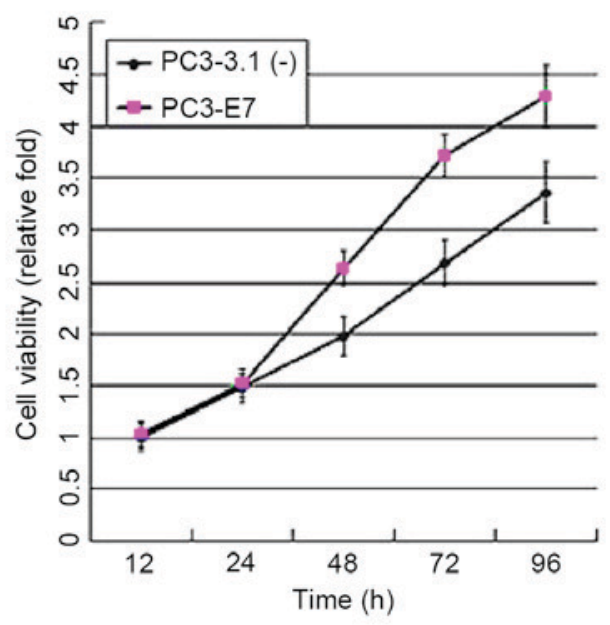

$\mathbf{F}$

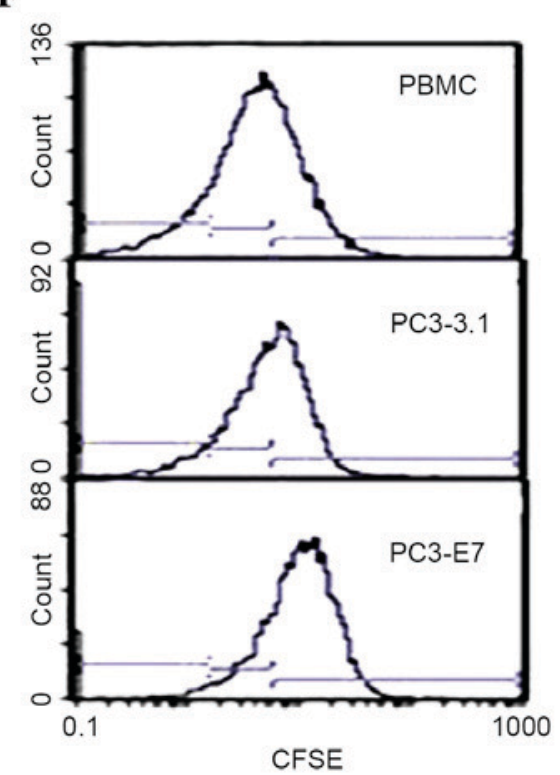

D

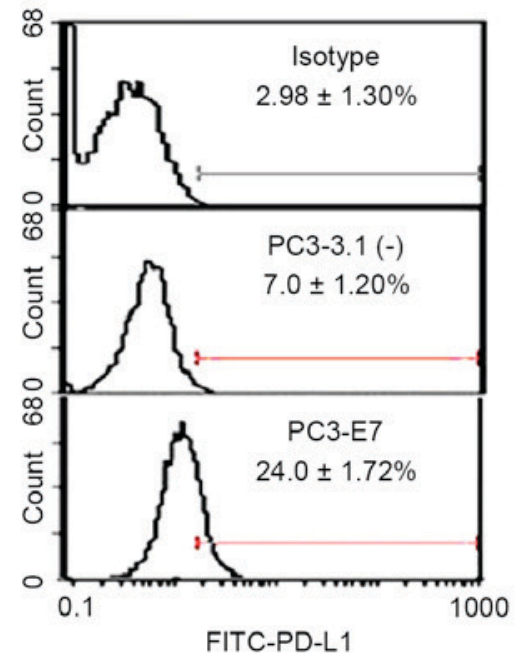

G

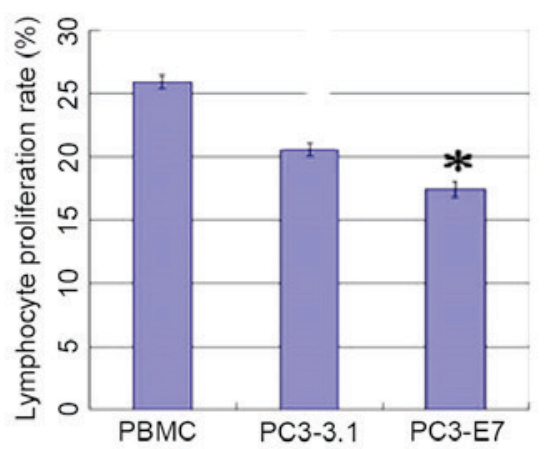

Figure 2. HPV16E7 upregulated PD-L1 expression in PC3 cells and inhibited lymphocyte proliferation. The recombinant plasmid [pcDNA3.1(-)-E7], or empty vector control [pcDNA3.1(-)], were and stably transfected into PC3 cells to generate positive PC3-E7 and PC3-3.1 clones, respectively. (A) HPV16E7 expression was detected in PC3-E7 clones by flow cytometry using fluorescein isothiocyanate-conjugated secondary antibodies. (B) DNA content analysis of the cell cycle in PC3-3.1 and PC3-E7 cells. (C) MTT analysis of cell viability. (D) The expression of PD-L1 was detected by flow cytometry analysis. (E) Reporter gene assay system analysis for the expression level of PD-L1 in PC3-E7 clones ("P<0.05 vs. PC3-3.1). (F) Flow cytometry analysis and (G) quantification of lymphocyte proliferation in the co-culture system ("P<0.05 vs. PC3-3.1). HPV16E7 human papillomavirus 16 E7 oncoprotein; PD-L1, programmed death-ligand 1; CFSE, carboxyfluorescein diacetate succimidyl ester; PBMCs, peripheral blood mononuclear cells.

Fig. 3C). Both flow cytometry and luciferase reporter system analysis demonstrated that PD-L1 expression was significantly reduced in CaSki-E7si cells $(\mathrm{P}=0.041$; Fig. 3D; $\mathrm{P}=0.027$; Fig. 3E). Following co-culture with lymphocytes, CaSki-E7si cells significantly stimulated lymphocyte proliferation when compared with CaSki-si-ctl cells $(\mathrm{P}=0.039 \mathrm{Fig}$. $3 \mathrm{~F}$ and $\mathrm{P}=0.035$; Fig. $3 \mathrm{G})$. According to the results presented in the current study, HPV16E7 overexpression in cervical cancer may lead to PD-L1 upregulation and mediate the escape of tumors from the immune system. 

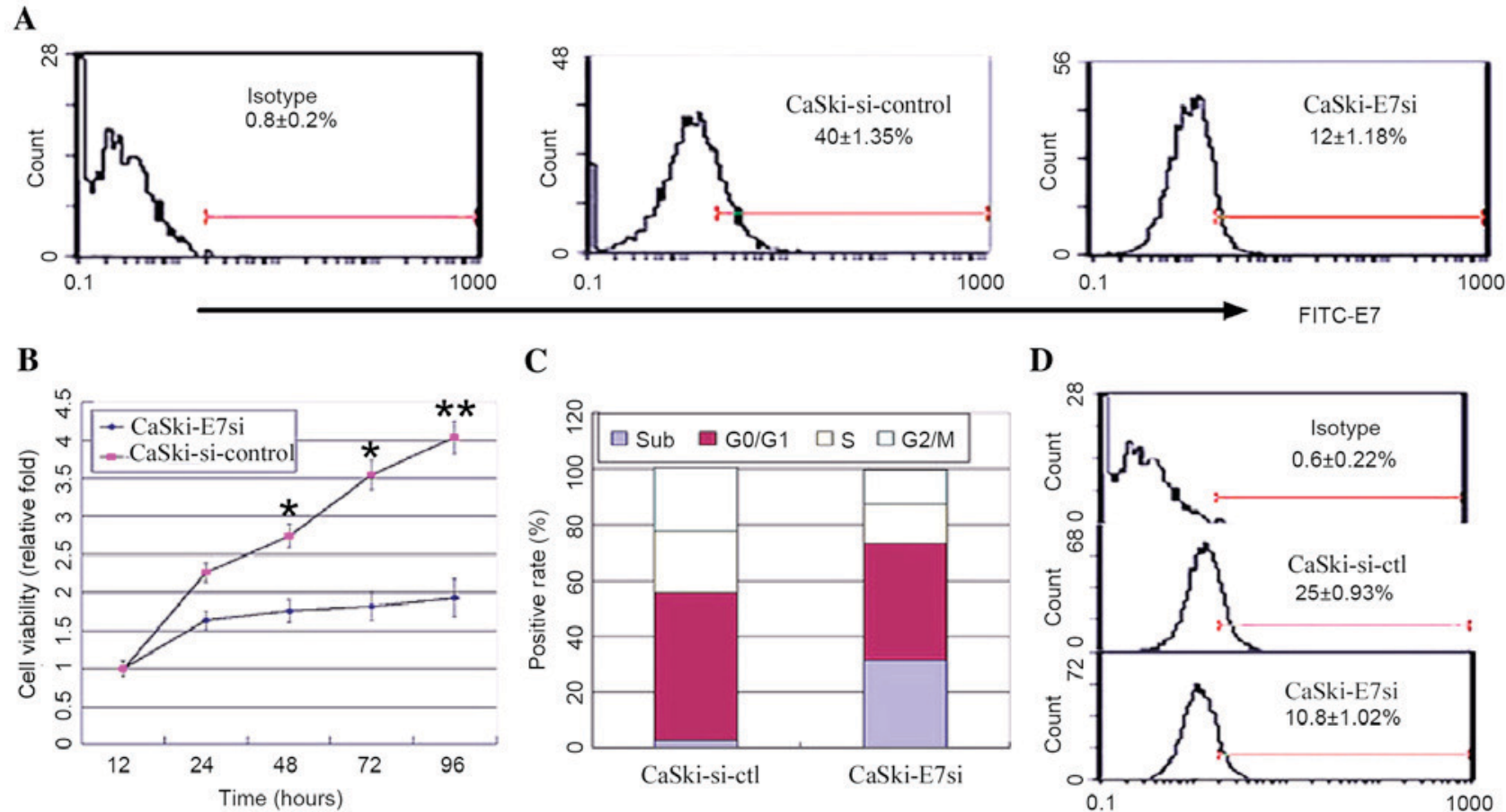

C

D
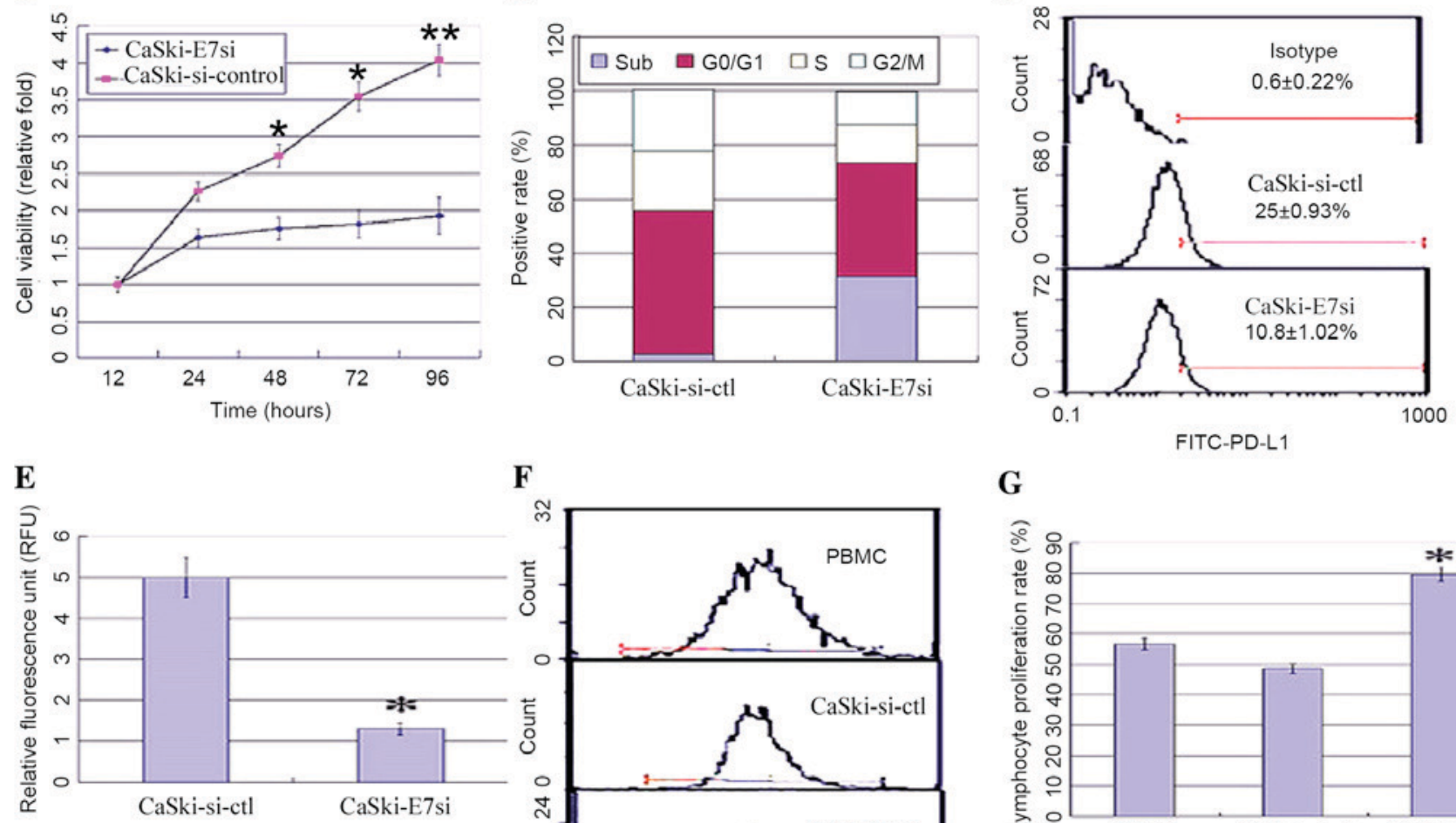

$\mathbf{F}$

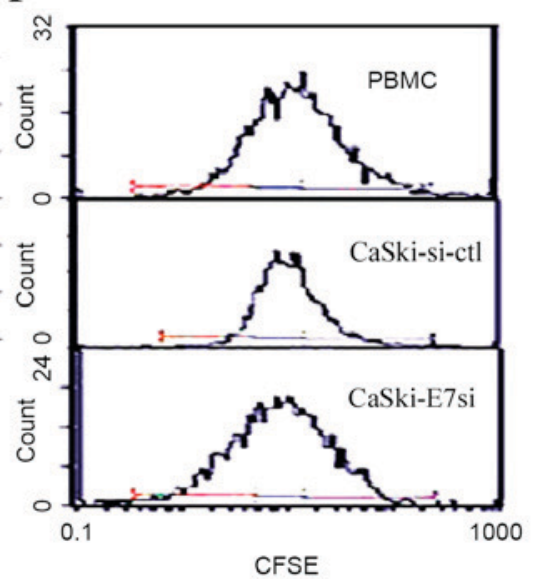

G

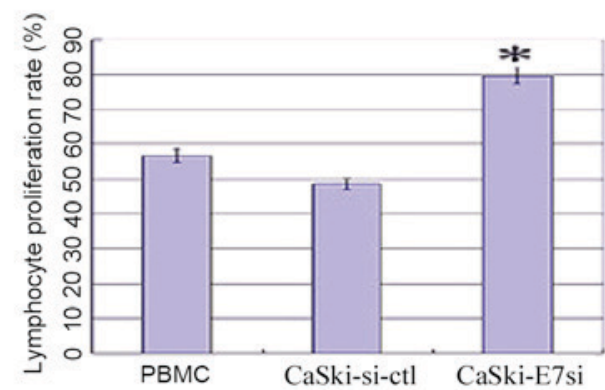

Figure 3. HPV16E7 knockdown downregulated PD-L1 in the CaSki cell line, and stimulated lymphocyte proliferation. pSilencer ${ }^{\mathrm{TM}}$ 2.1-U6-E7 or control vector pSilencer $^{\mathrm{TM}}$ 2.1-U6 siRNA vectors were stably transfected into CaSki cells to generate CaSki-E7si and CaSki-si-ctl cells, respectively. (A) Flow cytometry analysis of HPV16E7 expression in positive CaSki-E7si and CaSki-si-ctl clones. (B) MTT analysis of the viability of CaSki-E7si compared with controls. (C) DNA content analysis of the cell cycle in CaSki-si-ctl and CaSki-E7si cells. (D) Flow cytometry analysis of the expression of PD-L1. (E) Reporter gene assay system analysis of the expression levels of PD-L1 ("P<0.05 vs. CaSki-si-ctl). (F) Flow cytometry analysis and (G) quantification of the proliferation of CFSE-labeled PBMCs co-cultured with CaSki-E7si clones ("P<0.05 vs. CaSki-si-ctl). HPV16E7, human papillomavirus 16 E7 oncoprotein; PD-L1, programmed death-ligand 1; CFSE, carboxyfluorescein diacetate succimidyl ester; PBMCs, peripheral blood mononuclear cells.

Soluble PD-1 (sPD-1) promotes lymphocyte proliferation and cytotoxic activity. Due to the observation that HPV16E7 suppressed lymphocyte proliferation and cytotoxic activity potentially via upregulating PD-L1 expression in cervical cancer, the authors hypothesized that inhibition of the PD-L1/PD-1 signaling pathway may improve immunotherapy in cervical cancer. PD-1 expressed on lymphocytes binds to PD-L1 on tumor cells and mediates negative regulation of the immune response (13). Therefore, the recombinant plasmid sPD-1 was constructed and stably transfected into CaSki cells. Empty vector controls were denoted as CaSki-PIG. CaSki-sPD-1 cells were determined to express SPD-1 by RT-PCR and western blotting analyses (Fig. 4A). Following co-culture of lymphocytes with CaSki-sPD-1 cells, lymphocyte proliferation was significantly elevated when compared with CaSki-PIG cells ( $\mathrm{P}=0.038$; Fig. 4B). The activated lymphocytes exhibited significantly enhanced cytotoxic activity when they were co-cultured with CaSki-sPD-1 cells compared with CaSki-PIG cells ( $\mathrm{P}=0.042$; Fig. $4 \mathrm{C})$. These results demonstrated that inhibition of the PD-L1/PD-1 signaling pathway may partially restore the $\mathrm{T}$ cell response and enhance antitumor immunity.

\section{Discussion}

Persistent oncogenic HPV infection is a leading cause of cervical cancer (14). Viral oncoproteins E6 and E7 serve important roles in the initiation and malignant progression (15). 


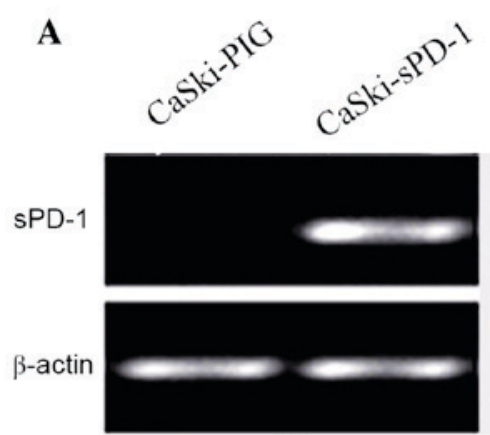

$\mathrm{A} 1$

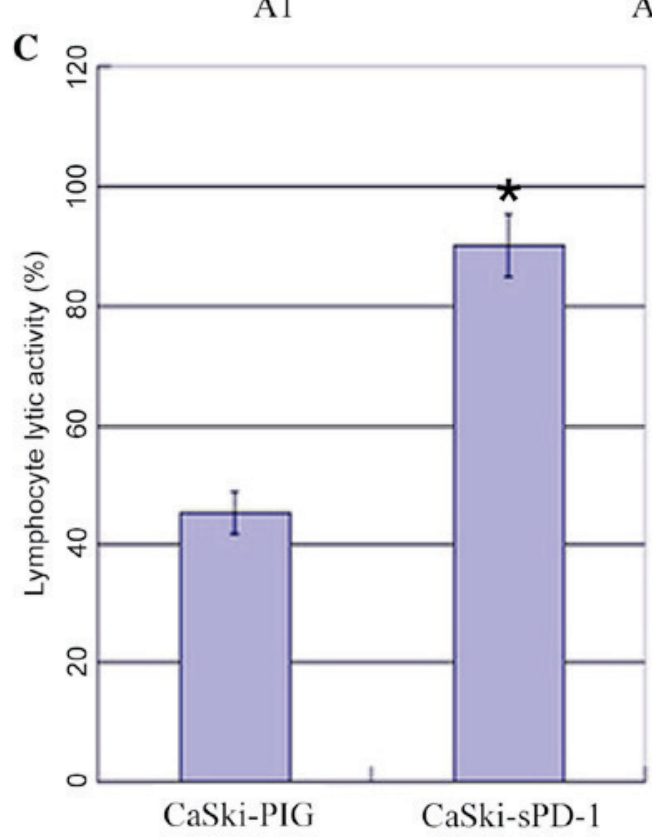

B

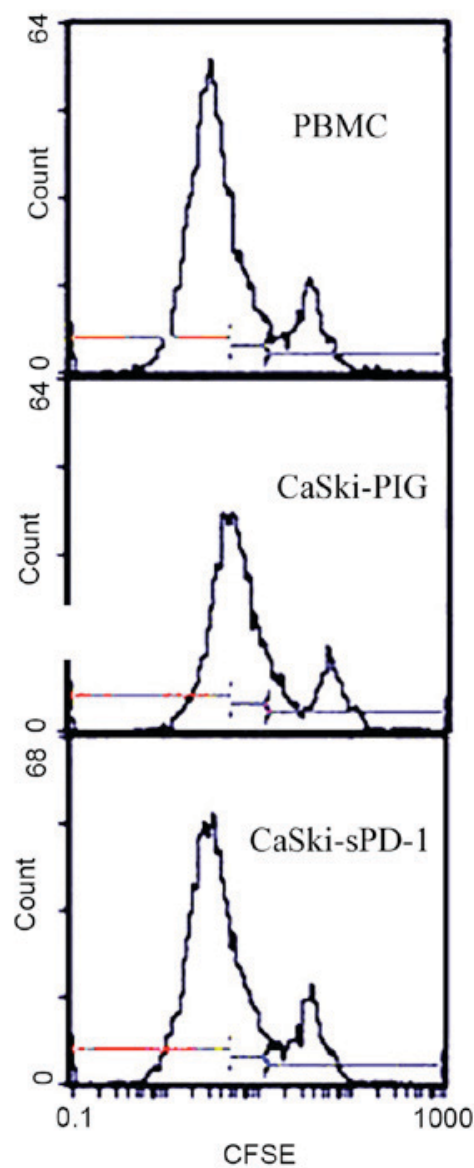

Figure 4. sPD-1 promotes lymphocyte proliferation and cytotoxic activities in CaSki cells expressing human papillomavirus 16 E7 oncoprotein. The recombinant MSCVPIG-sPD-1 and empty vector control plasmids were stably transfected into CaSki cells to produce CaSki-sPD-1 and CaSki-PIG clones, respectively. Positive clones exhibited a significant increase in lymphocyte proliferation and cytotoxic T lymphocyte activity. (A) Positive transfection clones were examined for the expression of PD-1 by (A1) reverse transcription-polymerase chain reaction and (A2) western blotting. (B) The proliferation of CFSE-labeled peripheral blood mononuclear cells, which were co-cultured with CaSki-sPD-1 and CaSki-PIG cells, was detected by flow cytometry. (C) Cytotoxicity assay for lymphocytes co-cultured with positive CaSki-sPD-1 and CaSki-PIG transfection clones ( $\mathrm{P}<0.05)$. sPD-1, soluble programmed death-1; PD-1, programmed death 1 ; CFSE; carboxyfluorescein diacetate succimidyl ester.

In the present study, HPV16E7 expression in normal and cancerous cervical tissues was examined. Higher levels of HPV16E7 expression were observed in cervical cancer tissues, when compared with normal cervical tissues. In addition, the study demonstrated that HPV16E7 promoted cell cycle progression and cell growth, whilst HPV16E7 knockdown increased sub G1 percentage which would indicate apoptosis of CaSki cells (Fig. 3C). The results indicated that the conversion from normal cervical epithelial cells to cervical cancer cells is a gradual process and that E7 expression level is positively correlated with cell transformation and tumor progression (16).

HPVs perturb cell growth, apoptosis and differentiation, however, they are additionally involved in affecting host anti-virus/tumor immune responses (17). Previous studies have reported that HPVE7 affects the innate immune response by downregulating the expression of interferon-responsive genes, which affects activation of the subsequent adaptive immune response $(18,19)$. Lymphocyte-mediated cytotoxicity involving cytotoxic $\mathrm{T}$ lymphocytes is the most effective mechanism for the control and clearance of viral infections $(20,21)$. In the current study, PC3-E7 or CaSki-E7si cells were co-cultured with PBMCs, and the results demonstrated that HPV16E7 modulated lymphocyte proliferation and cytotoxic activity. This suggested that HPV16E7 may induce HPV-infected cervical epithelial cells to circumvent the host immune response, however the mechanisms involved remain elusive.

$\mathrm{T}$ lymphocyte activation requires two signals, the recognition of a peptide (presented by major histocompatibility complex molecules on APCs) by a T cell receptor, and the second signal provided by co-stimulatory molecules (22). The balance of positive and negative signals is of central importance in maximizing the ability of the adaptive immune response to defend the host and/or maintain auto-tolerance. One of the previously identified $\mathrm{T}$ lymphocyte inhibitory molecules is PD-L1 (23). PD-L1 on APCs binds to its receptor PD-1 on activated $\mathrm{T}$ lymphocytes, resulting in inhibition of $\mathrm{T}$ lymphocyte activity $(8,9)$. Aberrant high expression of PD-L1 is frequent in various tumor tissues and is correlated with tumor progression (10,11). Fife and Pauken (24) demonstrated that, during 
chronic viral infections and cancer, PD-1-expressed on the $\mathrm{T}$ cell membrane surface encountered PD-L1 on infected or tumor cells and are associated with functional exhaustion of virus-specific $\mathrm{CD} 8^{+} \mathrm{T}$ cells. The results of the present study demonstrated that PD-L1 expression in cervical cancer tissues was significantly higher when compared with that in normal control tissues. A positive correlation between HPVE7 and PD-L1 expression was observed in cervical cancer tissues and the cell lines. Using a luciferase assay, it was demonstrated that the increased expression of PD-L1 was mediated by HPV16E7 in cervical cancer cells or cells overexpressing E7. These results suggest that HPV16E7 may inhibit lymphocyte proliferation and cytotoxic activity by upregulating PD-L1, thus resulting in immune escape of tumor cells.

Increased PD-L1/PD-1 expression on the cell membrane surface is the main cause of lymphocyte dysfunction during chronic HPV infection, and inhibiting this pathway may be beneficial to restore the function of tumor infiltrating lymphocytes $(25,26)$. Blockade of PD-1 using the specific anti-PD-1 antibody in vivo has been demonstrated to restore the function of virus-specific $\mathrm{CD} 8^{+} \mathrm{T}$ cells, which leads to enhanced inflammatory cytokines and viral clearance $(27,28)$. In conclusion, overexpressed PD-L1 was positively correlated with HPV16E7 in cervical cancer cells, which itself may have been responsible for lymphocyte dysfunction. In the present study, sPD-1-modified CaSki cells were co-cultured with PBMCs, which significantly elevated lymphocyte proliferation and cytotoxic activity. These results indicated that SPD-1 may restore lymphocyte function by inhibiting the PD-L1/PD-1 pathway, and provides a novel insight into immunotherapeutic approaches for the treatment of cervical cancer.

\section{Acknowledgements}

The present study was supported by the Major Research Plan of the Hubei Province Natural Science Foundation of China (grant no. 2012FFA086) and Hubei Province's Youth Science and Technology Innovation Team (grant no. T201203).

\section{References}

1. Parkin DM, Bray F, Ferlay J and Pisani P: Global cancer statistics, 2002. CA Cancer J Clin 55: 74-108, 2005.

2. Parkin DM and Bray F: Chapter 2: The burden of HPV-related cancers. Vaccine 24 (Suppl 3): S11-S25, 2006.

3. Scheffner M, Werness BA, Huibregtse JM, Levine AJ and Howley PM: The E6 oncoprotein encoded by human papillomavirus types 16 and 18 promotes the degradation of $\mathrm{p} 53$. Cell 63: 1129-1136, 1990.

4. Dyson N, Howley PM, Münger K and Harlow E: The human papilloma virus-16 E7 oncoprotein is able to bind to the retinoblastoma gene product. Science 243: 934-937, 1989.

5. Rosenberg SA, Yang JC and Restifo NP: Cancer immunotherapy: Moving beyond current vaccines. Nat Med 10: 909-915, 2004.

6. Dudley ME and Rosenberg SA: Adoptive-cell-transfer therapy for the treatment of patients with cancer. Nat Rev Cancer 3: 666-675, 2003

7. Postow MA, Callahan MK and Wolchok JD: Immune checkpoint blockade in cancer therapy. J Clin Oncol 33: 1974-1982, 2015.

8. Selenko-Gebauer N, Majdic O, Szekeres A, Höfler G, Guthann E, Korthäuer U, Zlabinger G, Steinberger P, Pickl WF, Stockinger H, et al: B7-H1 (programmed death-1 ligand) on dendritic cells is involved in the induction and maintenance of $\mathrm{T}$ cell anergy. J Immunol 170: 3637-3644, 2003.
9. Dong H, Strome SE, Salomao DR, Tamura H, Hirano F, Flies DB, Roche PC, Lu J, Zhu G, Tamada K, et al: Tumor-associated B7-H1 promotes T-cell apoptosis: A potential mechanism of immune evasion. Nat Med 8: 793-800, 2002.

10. Zang X and Allison JP: The B7 family and cancer therapy: Costimulation and coinhibition. Clin Cancer Res 13: 5271-5279, 2007.

11. Hino R, Kabashima K, Kato Y, Yaqi H, Nakamura M, Honjo T, Okazaki T and Tokura Y: Tumor cell expression of programmed cell death-1 Ligand 1 Is a prognostic factor for malignant melanoma. Cancer 116: 1757-1766, 2010.

12. Tavassoli FA and Devilee P: World Health Organization classification of tumours. Pathology and Genetics of the breast an female genital organs. Lyon, IARC Press, 2003.

13. Dong H, Strome SE, Salomao DR, Tamura H, Hirano F, Files DB, Roche PC, Lu J, Zhu G, Tamada K, et al: Tumor-associated B7-H1 promotes T-cell apoptosis: A potential mechanism of immune evasion. Nat Med 8: 793-800, 2002.

14. Muñoz N: Human papillomavirus and cancer: The epidemiological evidence. J Clin Virol 19: 1-5, 2000.

15. Isaacson Wechsler E, Wang Q, Roberts I, Pagliarulo E, Jackson D, Untersperger C, Coleman N, Griffin H and Doorbar J: Reconstruction of human papillomavirus type 16-mediated early-stage neoplasia implicates E6/E7 deregulation and the loss of contact inhibition in neoplastic progression. J Virol 86: 6358-6364, 2012.

16. Chen J, Xue Y, Poidinger M, Lim T, Chew SH, Pang CL, Abastado JP and Thierry F: Mapping of HPV transcripts in four human cervical lesions using RNAseq suggests quantitative rearrangements during carcinogenic progression. Virology 462-463: 14-24, 2014.

17. Bahrami AA, Ghaeni A, Tabarraei A, Sajadian A, Gorji A and Soleimanjahi H: DNA vaccine encoding HPV-16 E7 with mutation in L-Y-C-E pRb-binding motif induces potent anti-tumor responses in mice. J Virol Methods 206: 12-18, 2014.

18. Chang YE and Laimins LA: Microarray analysis identifies interferon-inducible genes and Stat-1 as major transcriptional targets of human papillomavirus type 31. J Virol 74: 4174-4182, 2000.

19. Nees M, Geoghegan JM, Hyman T, Frank S, Miller L and Woodworth CD: Papillomavirus type 16 oncogenes downregulate expression of interferon-responsive genes and upregulate proliferation-associated and NF-kappaB-responsive genes in cervical keratinocytes. J Virol 75: 4283-4296, 2001.

20. Stanley MA: Immune responses to human papilloma viruses. Indian J Med Res 130: 266-276, 2009.

21. Bontkes HJ, de Gruijl TD, Walboomers JM, van den Muysenberg AJ, Gunther AW, Scheper RJ, Meijer CJ and Kummer JA: Assessment of cytotoxic T-lymphocyte phenotype using the specific markers granzyme B and TIA-1 in cervical neoplastic lesions. Br J Cancer 76: 1353-1360, 1997.

22. Flies DB, Sandler BJ, Sznol M and Chen L: Blockade of the B7-H1/PD-1 pathway for cancer immunotherapy. Yale J Biol Med 84: 409-421, 2011.

23. Keir ME, Liang SC, Guleria I, Latchman YE, Qipo A, Albacker LA, Koulmanda M, Freeman GJ, Sayegh MH and Sharpe AH: Tissue expression of PD-L1 mediates peripheral T cell tolerance. J Exp Med 203: 883-895, 2006.

24. Fife BT and Pauken KE: The role of the PD-1 pathway in autoimmunity and peripheral tolerance. Ann N Y Acad Sci 1217: 45-59, 2011.

25. Lyford-Pike S, Peng S, Young GD, Taube JM, Westra WH, Akpeng B, Bruno TC, Richmon JD, Wang H, Bishop JA, et al: Evidence for a role the PD-1: PD-L1 pathway in immune resistance of HPV-associated head and neck squamous cell carcinoma. Cancer Res 73: 1733-1741, 2013.

26. Ukpo OC, Thorstad WL and Lewis JS Jr: B7-H1 expression model for immune evasion in human papillomavirus-related oropharyngeal squamous cell carcinoma. Head Neck Pathol 7: 113-121, 2013.

27. Barber DL, Wherry EJ, Masopust D, Zhu B, Allison JP, Sharpe AH, Freeman GJ and Ahmed R: Restoring function in exhausted CD8 T cells during chronic viral infection. Nature 439: 682-687, 2006.

28. Badoual C, Hans S, Merillon N, Van Ryswick C, Ravel P, Benhamouda N, Levionnois E, Nizard M, Si-Mohamed A, Besnier N, et al: PD-1 expression tumor-infiltrating T cells are a favorable prognostic biomarker in HPV-associated head and neck cancer. Cancer Res 73: 128-138, 2013. 